\title{
Measurement of Averaged Liquid Velocity Field around Large Bubbles Rising in Stagnant Water in Round Pipe Using UVP*
}

\author{
Hisato MINAGAWA**, Tsuyoshi FUKAZAWA***, Yoshiyuki NAKAZAWA***, \\ Satoshi YAMADA*** and Yoichi SHIOMI***
}

\begin{abstract}
An ultrasonic velocity profile monitor (UVP) measurement was performed to measure the average liquid velocity field around a large bubble rising in stagnant water in a round pipe of inner diameter $D=54 \mathrm{~mm}$ in order to obtain fundamental information for gas-liquid two-phase slug flows. Two ultrasonic transducers were set at different directions to obtain velocity vectors. The measured results are presented and compared with the results of some previous studies on the corresponding phenomena. In the liquid film near the bubble nose, the difference in bubble length does not affect the acceleration. The parameter $z / D$ is more dominant than $z$ itself, particularly when $z / D$ is less than unity. In the wake region, a large ring vortex is recognized. The upward velocity at the pipe axis agrees well with previously predicted results. The effect of bubble length on the vortex length is also discussed.
\end{abstract}

Key Words: Multiphase Flow, Bubble, Flow Measurements, Velocity Distribution, Vortex, Wake, Pipe Flow, Large Bubble, Average Velocity Field, Ultrasonic Measurements

\section{Introduction}

Gas-liquid two-phase slug flows in vertical pipes are frequently encountered in industrial pipelines, chemical and nuclear reactors and other fluid machineries ${ }^{(1)}$. The flow is characterized by a series of large bullet-shaped bubbles, or Taylor bubbles, which take up almost all the pipe area, and liquid slugs which contain many small bubbles. Their microscopic flow fields, however, have not been sufficiently clarified. Therefore, even the frictional pressure drop, which is necessary to design various types of equipment, has been obtained only under limited flow

* Received 15th June, 2006 (No. 05-0444). Japanese Original: Trans. Jpn. Soc. Mech. Eng., Vol.72, No.714, B (2006), pp.345-352 (Received 19th April, 2005)

** Department of Mechanical Systems Engineering, The University of Shiga Prefecture, 2500 Hassaka-cho, Hikone, Shiga 522-8533, Japan.

E-mail: minagawa@mech.usp.ac.jp

*** Division of Mechanical Systems Engineering, Engineering Graduate School, The University of Shiga Prefecture, 2500 Hassaka-cho, Hikone, Shiga 522-8533, Japan.

E-mail: satoshi@cont4.mech.usp.ac.jp

**** Department of Mechanical Systems Engineering, Ryukoku University, 1-5 Seta Ooe-cho Yokotani, Otsu, Shiga 520-2194, Japan.

E-mail: shiomi@rins.ryukoku.ac.jp conditions based on a precise flow model. This is due to the complex flow field of the two-phase slug flow, particularly around large bubbles. To determine the average velocity field around a large bubble in the slug flow, and to establish a more reasonable model of the flow, we are first required to know the velocity field around the large bubbles rising in a stagnant liquid, which is one of the simplified forms of large bubbles in slug flows in vertical pipes. Few studies on the average velocity field around large bubbles rising in a stagnant liquid have been reported. Therefore, in this study, the authors try to clarify the average velocity field around a large bubble using an ultrasonic velocity profiler (UVP). Here, the term "average" indicates the mean value of the velocity field for the plural flow fields around the same long large bubble. The pipe axis and radial components of the velocity vectors are simply averaged in this case.

Some of the existing studies on the measurement of the velocity field around a large bubble rising in a stagnant liquid include measurement using photochromic dye activation method by Kawaji et al. ${ }^{(2)}$, and those using PIV by Nogueira et al. ${ }^{(3)}$, van Hout et al. ${ }^{(4)}$, and Bugg and Saad $^{(5)}$. Tomiyama et al. ${ }^{(6)}$ also performed measurements using LDV, particularly to clarify the flow field in wakes behind the large bubbles. Among those studies, van Hout et al. ${ }^{(4)}$ and Tomiyama et al. ${ }^{(6)}$ used water as the liquid 
phase. However, no systematic data of the effect of bubble length is available for an air-water system. Moreover, in their researches, pipe diameter was limited to 19 32 mm. Consequently, we still require a systematic and precise data base on the flow field, particularly that in pipes with a larger diameter of about $50 \mathrm{~mm}$.

In this study, the UVP was used to measure the velocity field around a large bubble rising in stagnant water filling in a round vertical pipe of $54 \mathrm{~mm}$ I.D. The measurement covered the upper large bubble region, the liquid film around the large bubble, the wake below the large bubble, and the liquid slug which follows the wake.

The UVP is easy to operate, and it yields a velocity profile on one line instantaneously. It has, however, not been applied to the measurement of multiphase flows in a round pipe, except in the study on two-phase bubble flow $^{(7)}$. This is because one must obtain velocity profiles on two lines along different directions to obtain the velocity field. Moreover, it is difficult to apply ultrasonic methods to a round pipe with a large curvature because of the occurrence of reflection and refraction. However, once the measuring technique is fixed, the UVP will be one of the candidates for obtaining the velocity field around large bubbles systematically, because it is easy to operate, has effective time and space resolutions, yields velocity profiles on one line instantaneously, and is applicable to arbitrary liquid phase and pipe-wall materials including, of course, nontransparent liquids and walls.

\section{Nomenclature}

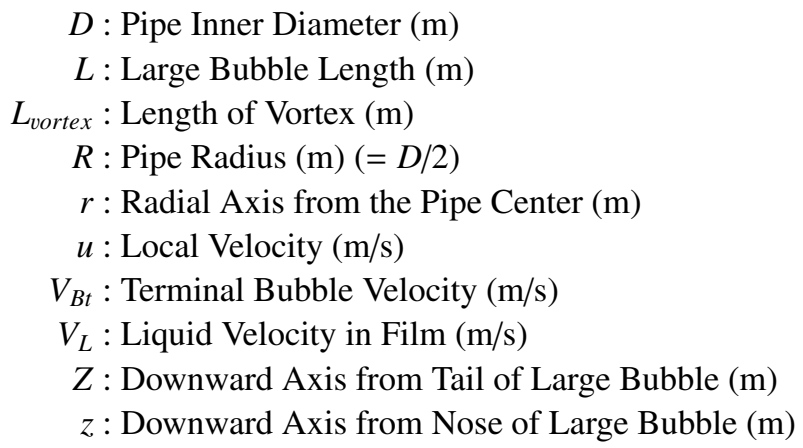

\section{Experimental Facilities}

\subsection{Experimental apparatus and procedure}

The UVP system used in this study emits a pulsed ultrasonic beam from its transducer. The ultrasonic echo from fine scattering particles premixed in the liquid phase is received by the same transducer. The measuring position is estimated from the time interval between pulse sending and reception and the sound velocity. The beam direction component of the local liquid velocity is estimated from the Doppler shift of the ultrasound. The Doppler shifts from numerous echoes from one beam are processed at the same time. Therefore, a velocity profile in the beam direction is obtained with one ultrasonic beam.

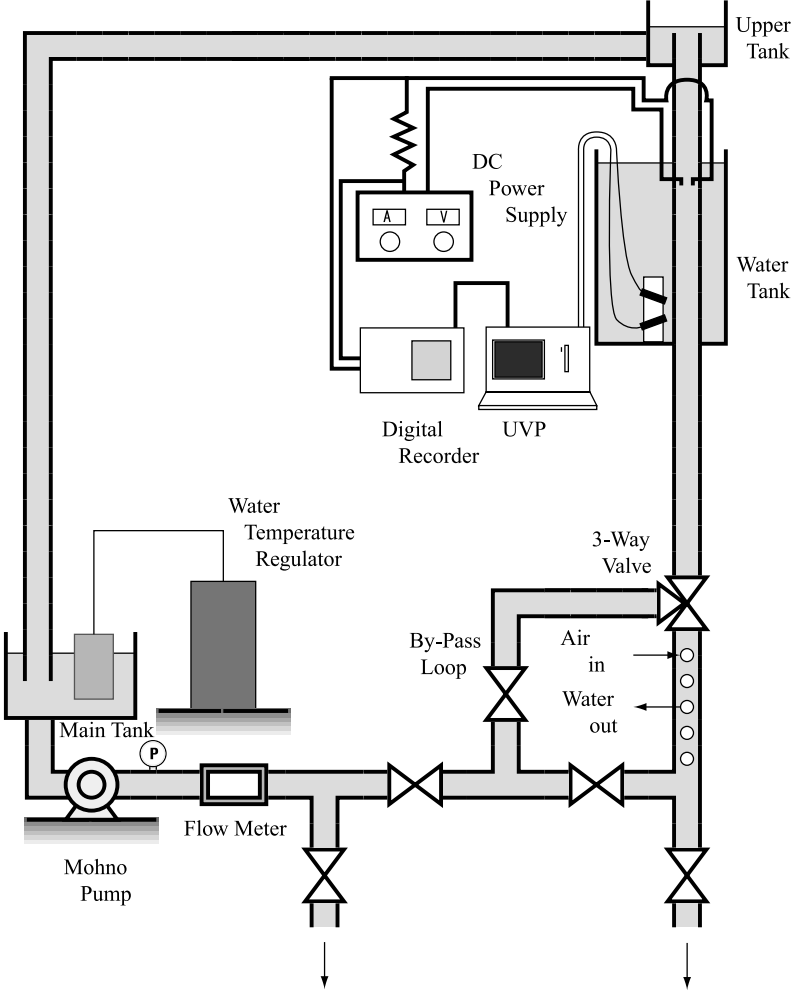

Fig. 1 Experimental apparatus

If we have two transducers which are set in different directions, we can calculate velocity vectors at a cross point. If we assume quasi-steady-state flow around a large bubble, the "average" velocity field can be obtained.

A schematic of the experimental apparatus is shown in Fig. 1. Tap water filled in the tank was supplied to the test section by a Mohno pump. Polyethylene particles of a median diameter of $160 \mu \mathrm{m}$ were premixed at $0.0375 \% \mathrm{wt}$ as the ultrasound-scattering particles. The temperature of the water was kept at $20 \pm 1^{\circ} \mathrm{C}$ by a regulator. When the test section was fully filled with water, the pump was stopped. The valves were closed, and air was stored in the pipe just under the 3-way valve by draining water from the pipe.

The test section consisted of a transparent acrylic vertical round pipe of $54 \mathrm{~mm}$ I.D. and about $3 \mathrm{~m}$ length. The details of the test section are shown in Fig. 2. Two ultrasonic transducers of the UVP (frequency: $4 \mathrm{MHz}$ ) were set outside of the test section at $2.3 \mathrm{~m}$ the 3 -way valve: one was inclined +20 degrees and the other -20 degrees from the horizontal line, as shown in Fig. 2. They were set in a water-filled tank, with the water surface covered with a styrene foam sheet to prevent noise caused by the shaking water surface. The measuring frequency of the UVP was $50 \mathrm{~Hz}$. A pair of electrodes (denoted by the large bubble sensor) was installed in the pipe $75 \mathrm{~mm}$ downstream of the transducers to determine the relationship between time and the position of the large bubble. Signals from the UVP and the large bubble sensor were recorded simultaneously on a digital recorder. When all the facilities were ready, 


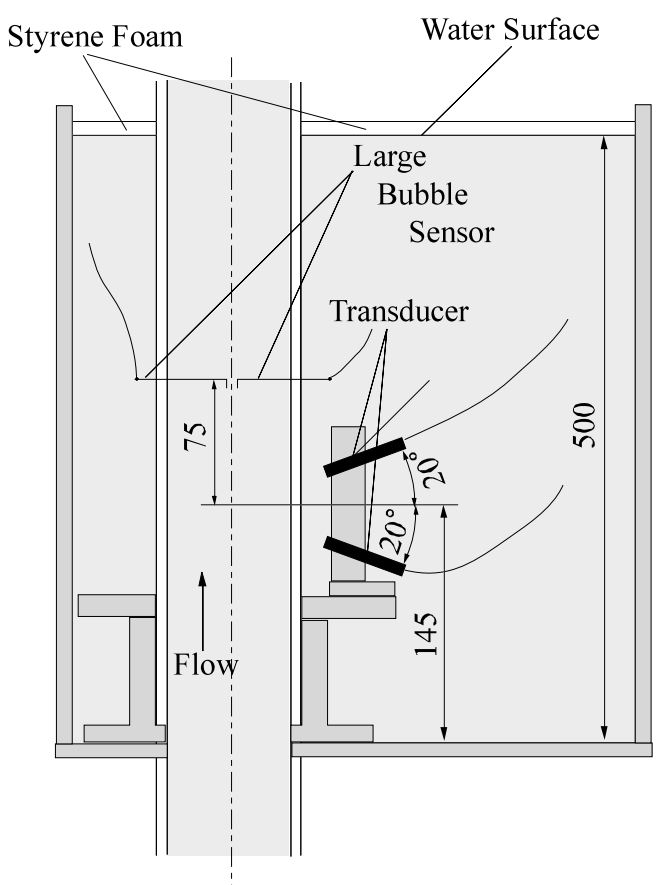

Fig. 2 Measuring section in detail

the 3-way valve was quickly opened; a large bubble rose up into the test section, and signals were recorded.

Velocity fields for about 20 large bubbles were measured for one bubble length in this study. Four groups of bubble length $(L=1.5 D, 2 D, 3 D$ and $4 D)$ were adopted to investigate the effect of large bubble length on the flow field around the large bubble. The average velocity was calculated in a cell of $5.38 \mathrm{~mm}$ in the flow direction and $0.695 \mathrm{~mm}$ in the radial direction; the velocity components in the cell were arithmetically averaged for each transducer direction. The average velocity vector in each cell was obtained through these two arithmetic averaged values. Since the ultrasonic beam diameter was $5 \mathrm{~mm}$ and the transducer inclinations were $\pm 20^{\circ}, 51 \%$ of the measuring volume overlapped radially adjacent cells. The measuring error may become large if the velocity gradient is steep. Therefore, in this study, we did not precisely discuss the velocity profile in the liquid film, in which the velocity gradient is assumed to be steep.

The pipe wall position was specified using the measured data for the liquid single-phase flow. Figure 3 exhibits the velocity profile of a liquid single-phase flow obtained with the same setup. Solid and open symbols are for the transducer with the elevation angle $\left(\theta=+20^{\circ}\right)$ and for the transducer with the depression angle $\left(\theta=-20^{\circ}\right)$, respectively. The solid line is an approximation using the $1 / 6$ th power law. Although the data are somewhat scattered in the far half of the transducer $(r / R>0)$, we can easily determine the wall position at the near side of the transducer $(r / R=-1)$. Data only in the near half of the transducer $(r / R<0)$ are processed in this study, and they

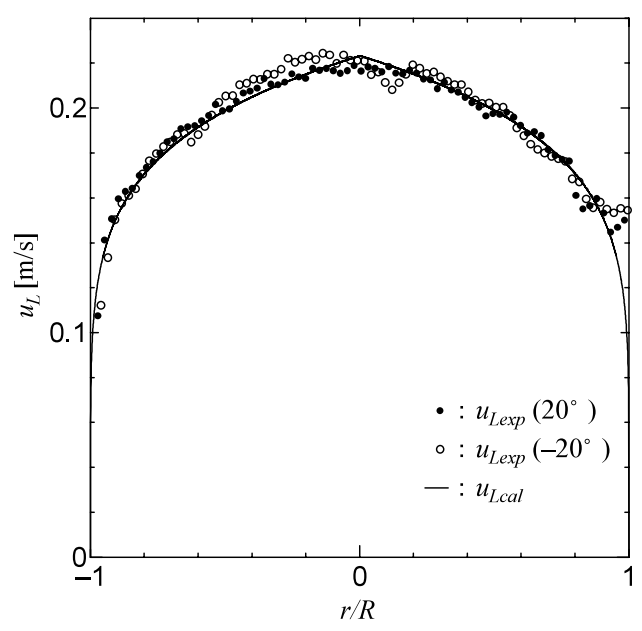

Fig. 3 Velocity profile of single phase flow

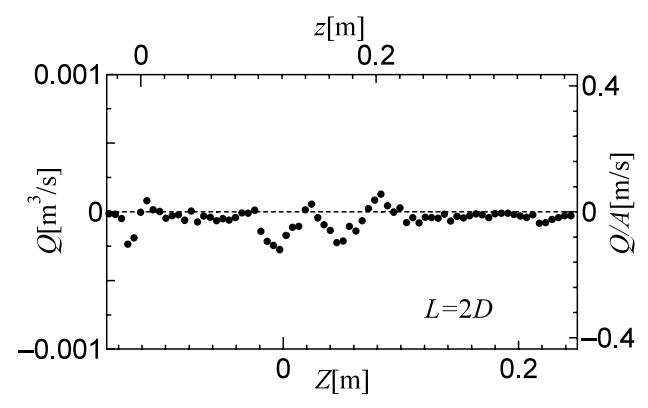

Fig. 4 Continuity relation

are folded back under the assumption of axial symmetry in this study.

\subsection{Shape of large bubble}

The shapes of large bubbles were obtained by measuring the film thickness around large bubbles using images from a digital video recorder. The effect of reflection due to the round pipe was compensated. As a result, the shapes of the large bubbles in this study are estimated with average relative error of $6.9 \%$ using the bubble shape function proposed by Nakahara et al. ${ }^{(8)}$

\subsection{Equation of continuity}

In order to confirm the uncertainty in measurement, the continuity relation was applied to the measured velocity. Figure 4 shows an example of the results for the large bubble length $L=2 D$. The left vertical axis of the figure shows the integrated value of the flow rate, $Q$, in the axial direction at each cross section. The right vertical axis shows the mean velocity, $Q / A$, where $A$ denotes the cross-sectional area. Because the flow system in this study is essentially static system, these values should ideally be zero. Although the flow rate data depart from the zero line to some degree around the large bubble tail $(Z=0)$, the average absolute value of velocity is not very large ( = $0.0291 \mathrm{~m} / \mathrm{s})$ compared with the large bubble rising velocity, $V_{B t}(=0.269 \mathrm{~m} / \mathrm{s})$. It can be confirmed that the UVP measurement system in this study can be used to measure the velocity field at the above-described accuracy. 


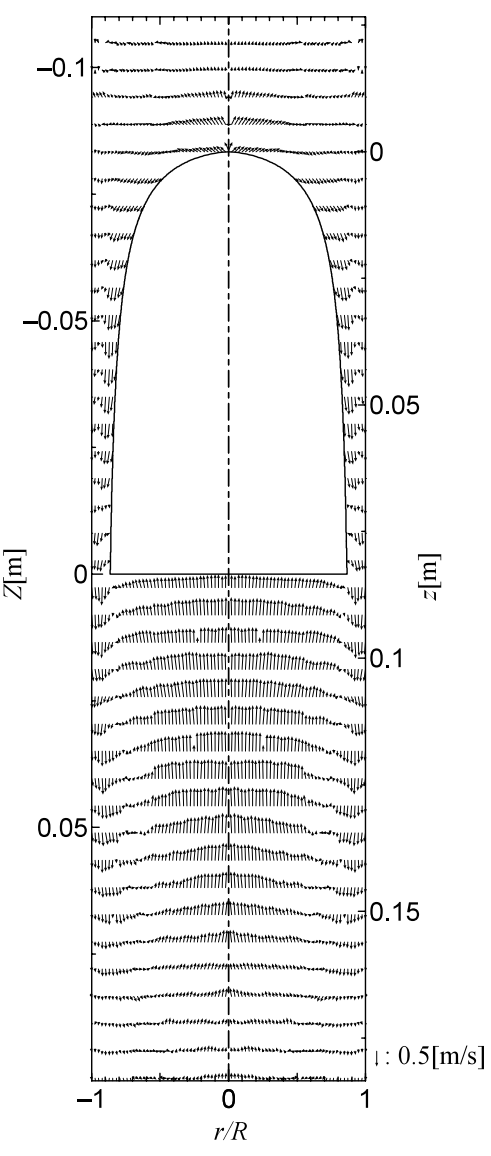

Fig. 5 Velocity field for $L=1.5 D$

\section{Experimental Results and Discussion}

\section{1 Outline of velocity field}

An example of the obtained result of the velocity field is presented in Fig. 5 for a whole slug unit with $L=1.5 D$. $Z$ denotes the downward distance from the tail of the large bubble and $z$ that from the nose. It is recognized that very low velocities are measured in the liquid phase above the large bubble. On the other hand, very high velocities are found in the liquid phase in the liquid film around and behind the large bubble. We can observe a downward accelerating flow in the liquid film, which penetrates the liquid bulk behind the large bubble near the wall and rapidly attenuates. When examining the liquid film velocity in detail, the velocity decreases once near the bubble tail $(Z \cong-0.01)$. This is because the bubble tail oscillates periodically under our experimental conditions. Hence it sometimes causes upward flow in the liquid film. Averaging the velocity for many large bubbles resulted in such a superficial deceleration. At the center of the pipe just below the large bubble, we can see large upward flows, which form a large ring vortex with the wall-side downward flow.

Below, the precise characteristics in each part of the slug unit are presented and discussed, considering the effect of large bubble length $L$.

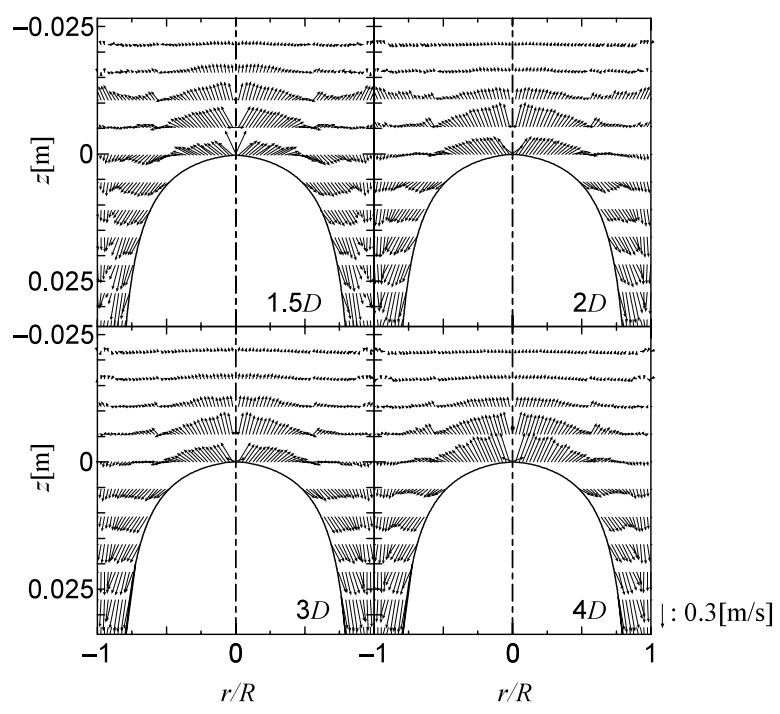

Fig. 6 Velocity field in the liquid phase in front of the large bubble

\subsection{Liquid phase above large bubble}

In order to clarify the flow field, we enlarged the velocity vector display in this region, as shown in Fig. 6, because in the liquid phase in front of the large bubble, only very low velocities are recognized. For all large bubble lengths, the effect of the rising large bubble is recognized to be approximately $0.020 \mathrm{~m} \cong D / 3$ from the bubble nose. van Hout et al. ${ }^{(4)}$ found that their corresponding length was nearly $0.0125 \mathrm{~m} \cong D / 2$ from the nose. In this affected region, the liquid near the pipe axis is lifted owing to the rising bubble nose, whereas, near the pipe wall, downward flow, which will flow into the liquid film around the large bubble, is recognized. There is another downward flow near the pipe wall from $z \cong 0$. These two downward flows join together in the film to form a complex flow pattern.

The effect of bubble length is essentially negligible for the velocity field in this area; even when the bubble length changes from $1.5 D$ to $4 D$, the velocity field remains unchanged. This is natural because the bubble rising velocity and bubble nose shape are identical for different bubble lengths, and this area is connected the area where the effect of pipe diameter is obvious, as will be described later (wake), only through the thin liquid film.

\subsection{Liquid film around large bubble}

The velocity profiles in the liquid film are shown and are compared with van Hout et al.'s ${ }^{(4)}$ and Kawaji et al.'s ${ }^{(2)}$ results in Fig. 7 (a) - (b). The plotted data, $V_{L f i l m}$, are obtained as the vertical downward component of the velocity vectors. In Fig. 7 (a), the symbol parameter is $z$. In this case, the velocities measured in this study are smaller than van Hout et al.'s and Kawaji et al.'s data for the same value of $z$. The difference is more marked when $z$ is smaller. Thus, even if the distance from the nose is the same, the liquid velocity in the film falls faster when $D$ is smaller.

Figure 7 (b) is a similar plot to that in Fig. 7 (a) with 


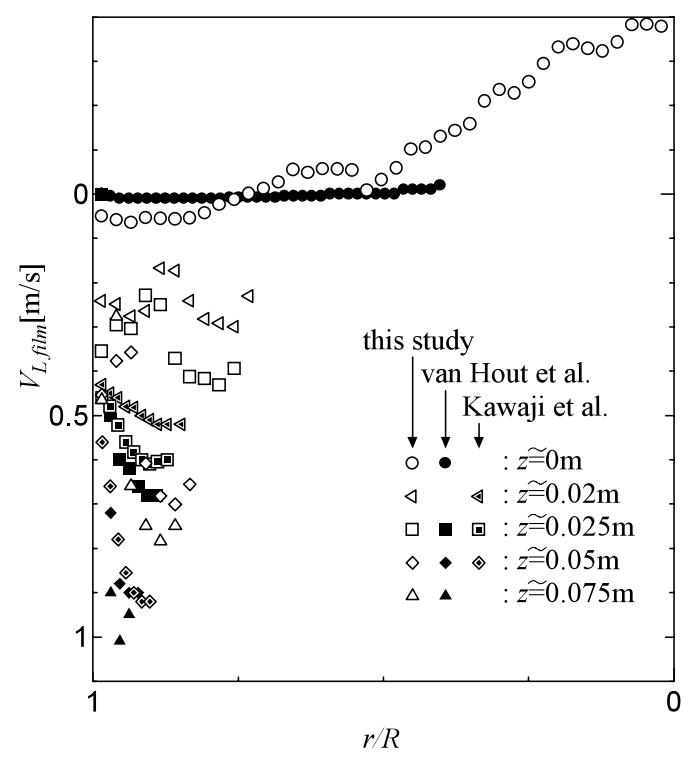

(a) $z$ as parameter

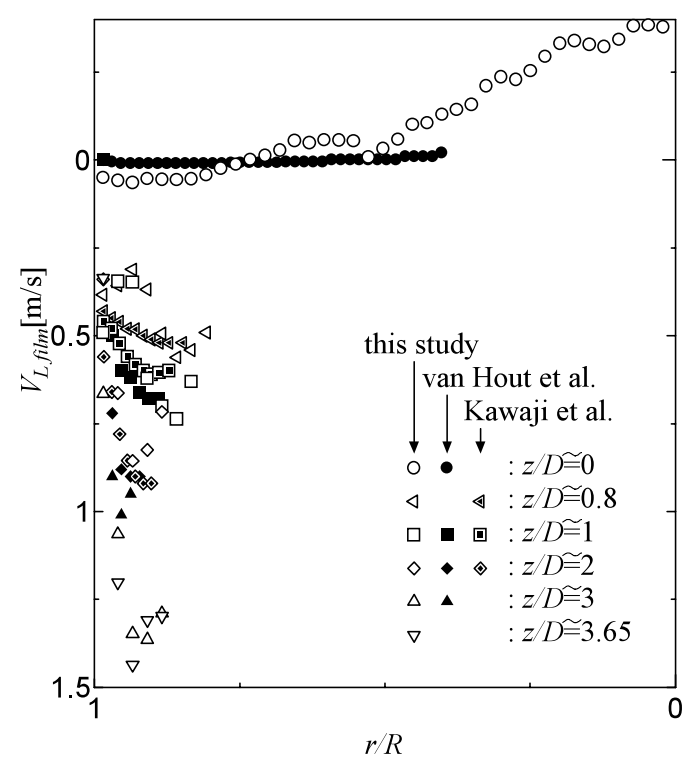

(b) $z / D$ as parameter

Fig. 7 Velocity profiles in the liquid film $(L=4 D)$

$z / D$ as a parameter instead of $z$. When the value of $z / D$ is smaller than unity, the velocity profiles in this study agree with those of the two existing studies to a certain degree. When $z / D$ exceeds unity, the liquid velocities in this study are larger than those in the other studies. This is probably because of the difference in pipe diameters. Although van Hout et al.'s and Kawaji et al.'s measurements were based on experiments using a pipe with diameter of approximately $25 \mathrm{~mm}$, our UVP measurement was performed for a $D=54 \mathrm{~mm}$ pipe. Thus, $z / D$ is more dominant than $z$ itself, particularly when $z / D$ is less than unity.

The maximum downward velocities, $V_{L \max }$, in the liquid film are plotted against $z$ in Fig. 8 (a), and against $z / D$ in Fig. 8 (b) again with van Hout et al.'s ${ }^{(4)}$ and Kawaji et

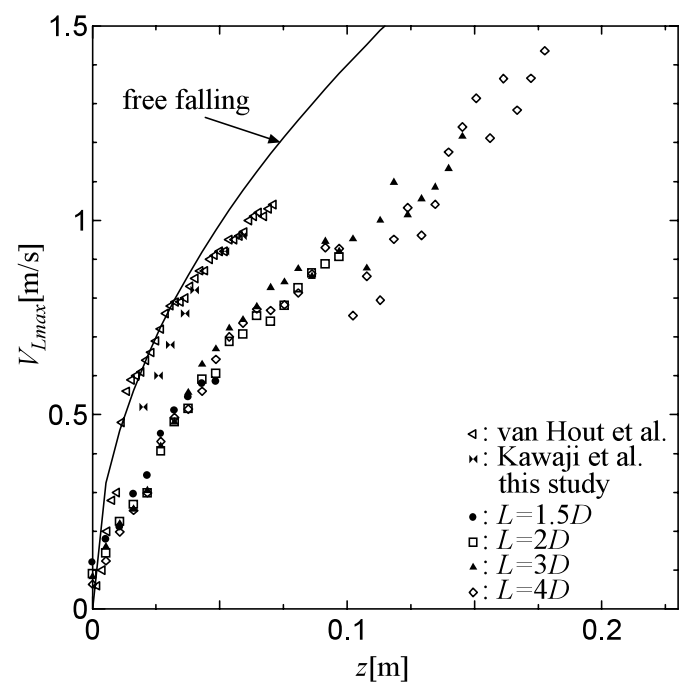

(a) $z-V_{\operatorname{Lmax}}$

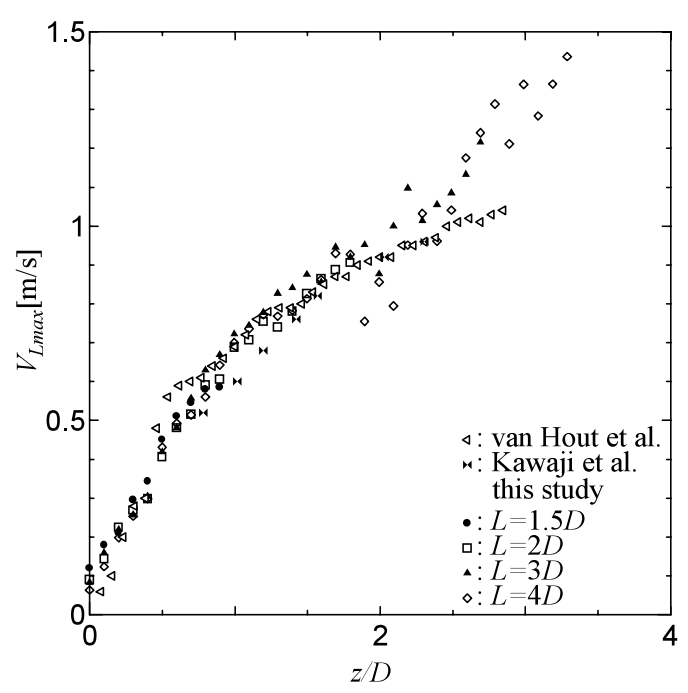

(b) $z / D-V_{\text {Lmax }}$

Fig. 8 Maximum downward velocity in the liquid film

al.'s ${ }^{(2)}$ data. The liquid downward velocity increases as it travels downward. As a result, the film thickness gradually becomes thin. However, the accelerations are somewhat lower than free-fall velocity and those in existing studies for smaller $D$, in which $V_{L m a x}$ almost agrees with free-fall velocity until about $z=0.3 \mathrm{~m}$. This is probably due to the larger diameter in this study. The large bubble rising velocity is higher in this study $(0.269 \mathrm{~m} / \mathrm{s})$ than in the other studies (about $0.17 \mathrm{~m} / \mathrm{s}$ ). The upward flow described in section 4.2 is therefore more powerful, which may decelerate the downward velocity, and the acceleration superficially becomes somewhat lower than that of free-fall. The difference in bubble length does not affect the acceleration, but it affects the velocity of the liquid penetrating into the wake region as an annular jet; the longer the bubble, the faster the liquid flow.

In Fig. 8 (b), $V_{\text {Lmax }}$ data in all studies agree with each other from the bubble nose to about $z / D=2$. As a re- 
sult, $z / D$ seems more dominant than $z$ itself, as shown in Fig. 7 (b). The data, however, depart from van Hout et al.'s ${ }^{(4)}$ data after the value of $z / D$ exceeds 2 .

\section{4 Wake below large bubble}

The velocity fields in the wake just below the large bubble are shown in Fig. 9(a)-(d) for each large bubble length. As mentioned above, a downward accelerating flow in the liquid film around the large bubble penetrates the liquid bulk, called the wake, behind the large bubble near the wall. It causes a downward flow in the wake near the pipe wall. The velocity of the downward flow depends on the large bubble length; the longer the bubble, the faster the flow. Around the pipe axis, on the other hand, an upward flow appears following the large bubble. These two flows form a large ring vortex near the large bubble tail, as shown in Fig. 9. van Hout et al. ${ }^{(4)}$ confirmed that a large vortex of $L_{\text {vortex }}=2 D \cong 0.05 \mathrm{~m}$ appears from $Z=0$, and the second weaker counter-rotating vortex appears at $Z=2 D \sim 4 D$ under their experimental condition of $D \cong 25 \mathrm{~mm}$. In this study, the second counter-rotating vortex was not recognized.

The longitudinal length of the ring measured from the stream line, $L_{\text {vortex }}$, is plotted versus $L / D$ in Fig. 10 . It has a maximum value at $L \cong 2 D$. Although the liquid velocity penetrating into the wake increases for $L=3 D$ and $4 D$, the value of $L_{\text {vortex }}$ decreases.

Figure 11 displays a plot of the measured results of the upward velocity at the pipe axis, $-u_{\text {Laxis }}$, for each large bubble length. The curved lines in the figure are the corresponding estimated results calculated by Tomiyama et al. ${ }^{(6)}$ The measured $-u_{\text {Laxis }}$ at the bubble tail $(Z=0)$ approximately agrees with the large bubble rising velocity, $V_{B t}$. It steeply increases and it has a maximum at around $Z=0.04$ to $0.06 \mathrm{~m}$. After that, it steeply decreases and falls below the value of $V_{B t}$ at around $Z=0.07$ to $0.09 \mathrm{~m}$. Subsequently, it approaches 0 asymptotically, although data are highly scattered. These characteristics agree well with results estimated by Tomiyama et al. ${ }^{(6)}$ both qualitatively and quantitatively. According to Tomiyama et al. ${ }^{(6)}$, the region where $-u_{\text {Laxis }}$ exceeds $V_{B t}$ is the "near-wake" region. The other region is the "far-wake" region. They suggested that the reason why $L_{v o r t e x}$ decreases with increasing $L / D$ when $L$ is longer than a certain value is that the vortexes become unsteady and are released periodically for a large L. Our measured results support their explanation. In order to clarify it more definitively, the standard deviation of the flow field is adopted.

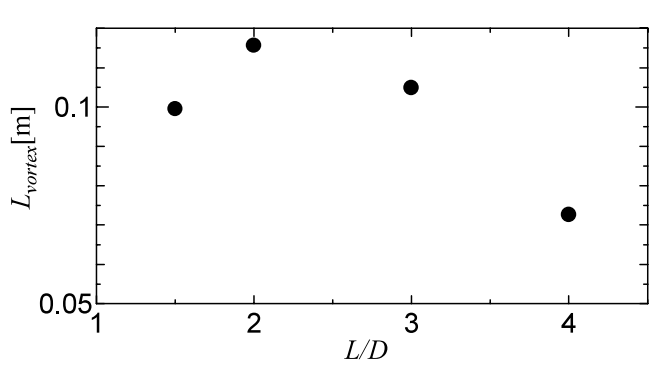

Fig. 10 Longitudinal length of ring vortex

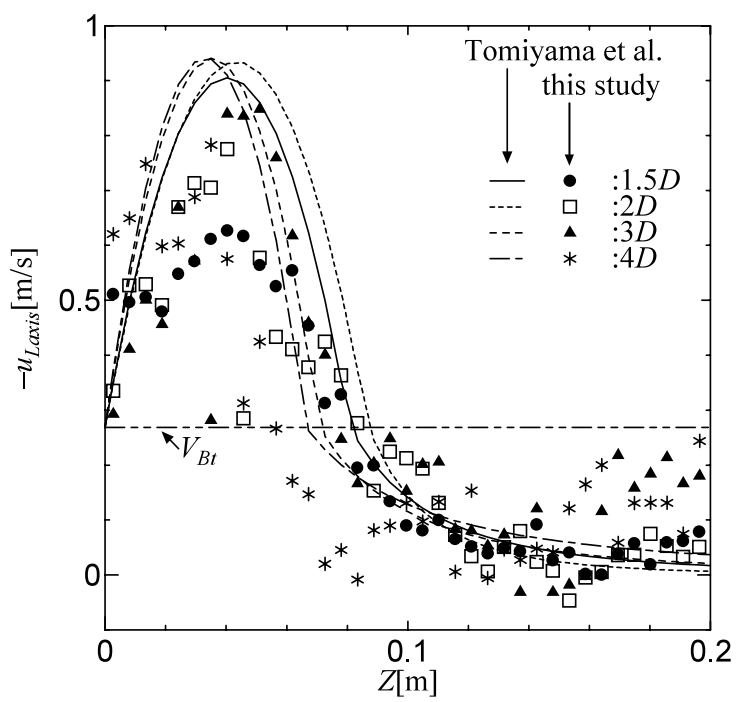

Fig. 11 Upward liquid velocity at the pipe axis

Fig. 9 Velocity field in the wake behind the large bubble 


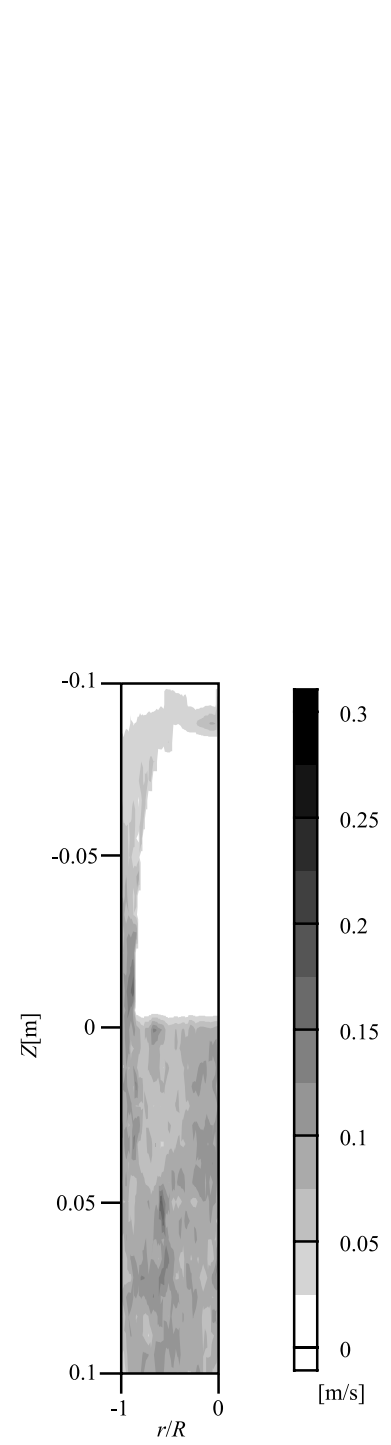

(a) $L=1.5 D$

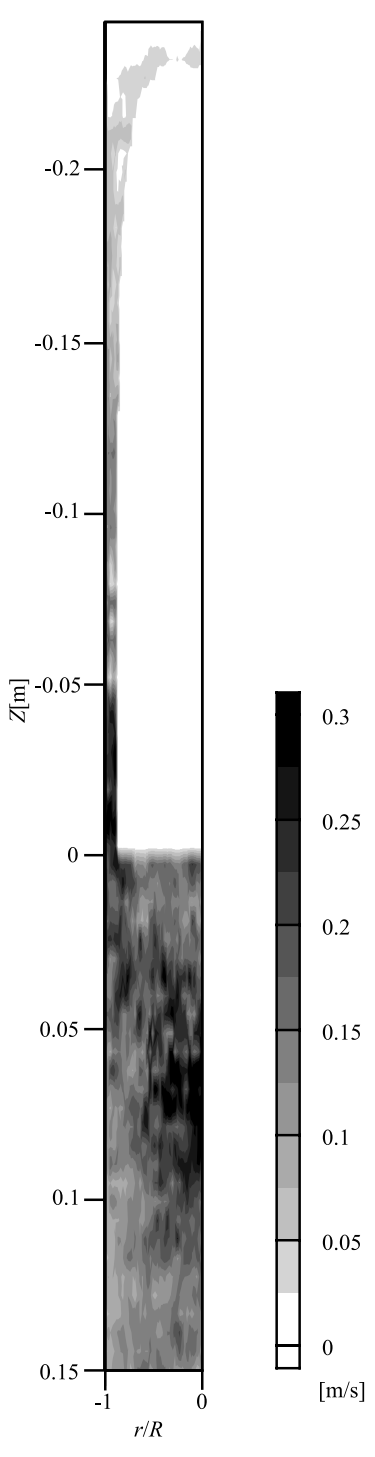

(b) $L=4 D$

Fig. 12 Standard deviation of liquid velocity

\subsection{Standard deviation of flow field}

Figure 12 shows the standard deviation of the vertical component of the liquid velocity in the flow field by the gradation sequence for a short large bubble, $L=1.5 \mathrm{D}$ in (a), and a long bubble, $L=4 D$ in (b). It is clearly confirmed that the standard deviation, particularly in the far wake region, is much larger when $L=4 D$. When the large bubble is long, the vortexes are released periodically from the near-wake to the far-wake region, which increases the standard deviation. Therefore, we can recognize the periodical release of vortexes when $L=4 D$.

\section{Conclusion}

Ultrasonic velocity profile monitor (UVP) measurements were performed to measure the average liquid velocity field around a large bubble rising in stagnant water in a round pipe of inner diameter $D=54 \mathrm{~mm}$ in order to obtain fundamental information for gas-liquid two-phase

slug flows. The measured results are presented not only for a whole large bubble system but for each part of the large bubble: the liquid phase in front of the large bubble, the liquid film around the large bubble, and the wake below the large bubble. The authors obtained the following findings.

(1) In the liquid phase in front of the large bubble, the liquid near the pipe axis is lifted owing to the rising bubble nose, whereas near the pipe wall, downward flow is recognized. There is another downward flow near the pipe wall from $z \cong 0$. These two downward flows join together in the film to form a complex flow pattern. The effect of bubble length is essentially negligible for the velocity field in this area.

(2) In the liquid film near the bubble nose, the parameter $z / D$ is more dominant than $z$ itself, particularly when $z / D$ is less than unity. The difference in the bubble length does not affect the acceleration, but it affects the velocity of liquid penetrating into the wake region as an annular jet; the longer the bubble, the faster the liquid flow.

(3) In the liquid phase behind the bubble tail or in the wake region, a large ring vortex is formed near the large bubble tail. The longitudinal length of the ring measured from the streamline has a maximum value at $L \cong 2 D$. Although the liquid velocity penetrating into the wake increases when $L=3 D$ and $4 D$, the longitudinal length of the ring decreases. According to Tomiyama et al. ${ }^{(6)}$, this is because when $L$ is longer than a certain value, vortexes become unsteady and are released periodically. Our measured results support their explanation. The standard deviation of the flow field also confirms this explanation.

\section{Acknowledgement}

We express our gratitude to Professor M. Aritomi and Dr. H. Kikura of Tokyo Institute of Technology for providing beneficial information on UVP measurements. We also thank the students Mr. M. Hirose and Mr. M. Ibuki, who performed the measurements in this study.

\section{References}

( 1 ) JSME, Handbook of Gas-Liquid Two-Phase Flow Technology, (in Japanese), (1989), Corona Pub.

(2 ) Kawaji, M., Dejesus, J.M. and Tudose, G., Investigation of Flow Structures in Vertical Slug Flow, Nuclear Engineering and Design, Vol.175 (1997), pp.37-48.

( 3 ) Nogueira, S., Riethmuller, M., Campos, J.B.L.M. and Pinto, A.M.F.R., Flow Patterns in the Wake of Taylor Bubbles Rising in Stagnant Liquid, Proc. 4th International Conference on Multiphase Flow, on CD-Rom, (2001).

(4) van Hout, R., Gulitski, A., Barnea, D. and Shemer, L., Experimental Investigation of the Velocity Field Induced by a Taylor Bubble Rising in Stagnant Water, International Journal of Multiphase Flow, Vol.28 (2002), pp.579-596. 
( 5 ) Bugg, J.D. and Saad, G.A., The Velocity Field around a Taylor Bubble Rising in a Stagnant Viscous Fluid: Numerical and Experimental Results, International Journal of Multiphase Flow, Vol.28 (2002), pp.791-803.

( 6 ) Tomiyama, A., Tamai, H. and Hosokawa, S., Velocity and Pressure Distributions around Large Bubbles Rising through a Vertical Pipe, Proc. 4th International Conference on Multiphase Flow, on CD-Rom, (2001).

( 7 ) Murakawa, H., Shigefuzi,M., Kikura, H. and Aritomi,
M., Bubbly Flow Measurement for Each Bubble Size Using Ultrasonic Doppler Method and Wire Mesh Tomography, Proc. 5th International Conference on Multiphase Flow, on CD-Rom, (2004).

( 8 ) Nakahara, Y., Morita, G. and Tomiyama, A., Study on Shape and Terminal Rising Velocity of Single Bubbles Moving in a Vertical Pipe, Proc. JSMF Annual Meeting 2000, (in Japanese), (2000), pp.175-176. 